\title{
ATLAS online data quality monitoring
}

\author{
Cristobal Cuenca Almenar, Yale \\ Alina Corso-Radu, Serguei Kolos, Kevin Slagle, Anyes Taffard, UCI \\ Haleh Hadavand, Yuriy Ilchenko, SMU
}

12th Topical Seminar on Innovative Particle and Radiation Detectors, Siena, June 7th-10th 2010

\section{ATLAS Data Acquisition system}

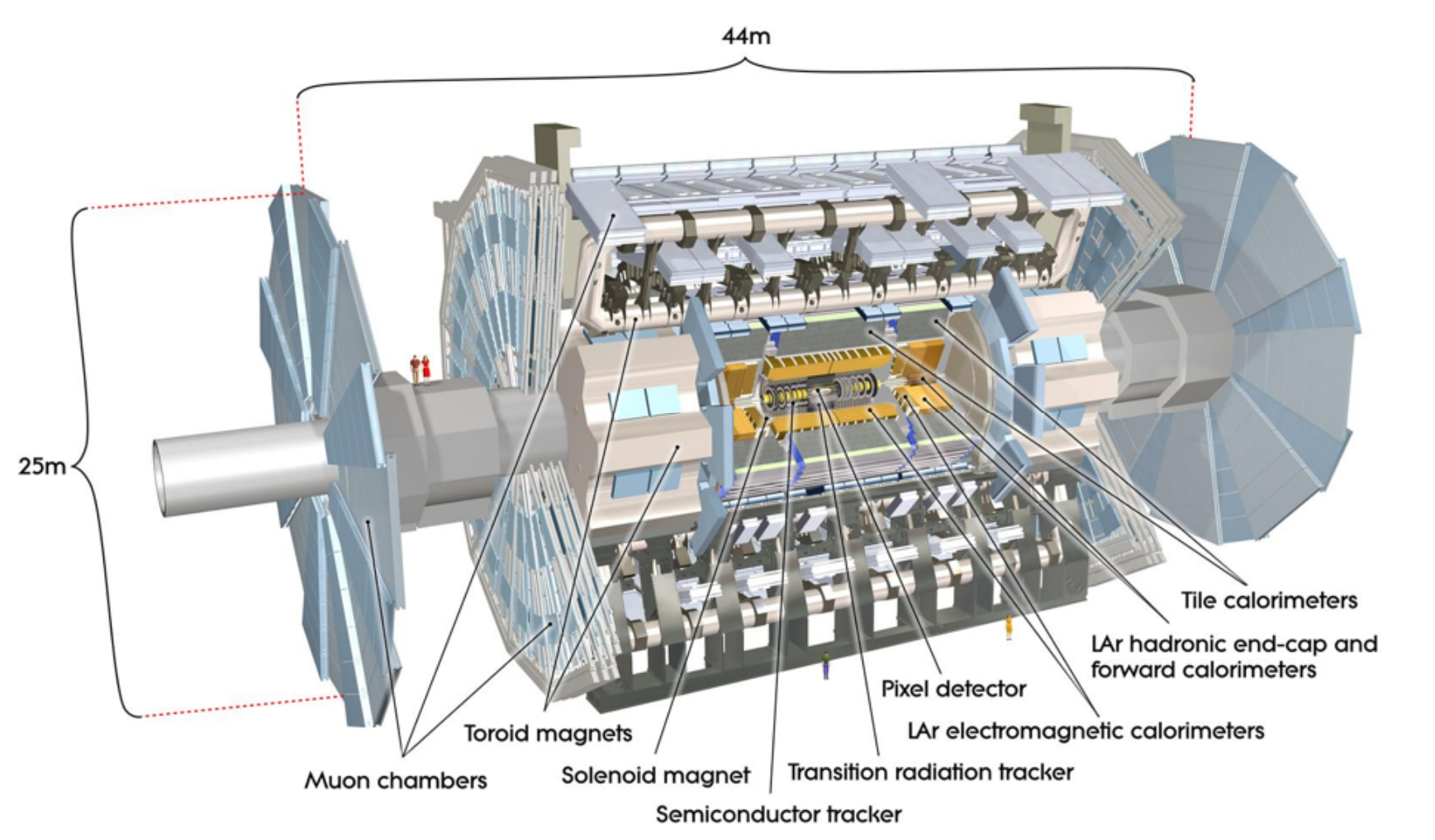

\section{The Data Acquisition system}

Data acquisition is based on a three-level trigger architecture. The goal is to achieve a final rate of $200 \mathrm{~Hz}$, from the $40 \mathrm{MHz}$ protonproton collision rate.

Data Flow is responsible for collecting data fragments, serving them to trigger processors and sending them to mass storage. Online data quality monitoring analyzes data from all subsystems at all stages of Data Flow

\section{The ATLAS detector}

The ATLAS experiment is one of the generic detectors designed and built to record the output of the proton-proton collisions provided by the Large Hadron Colider at CERN, Geneva.

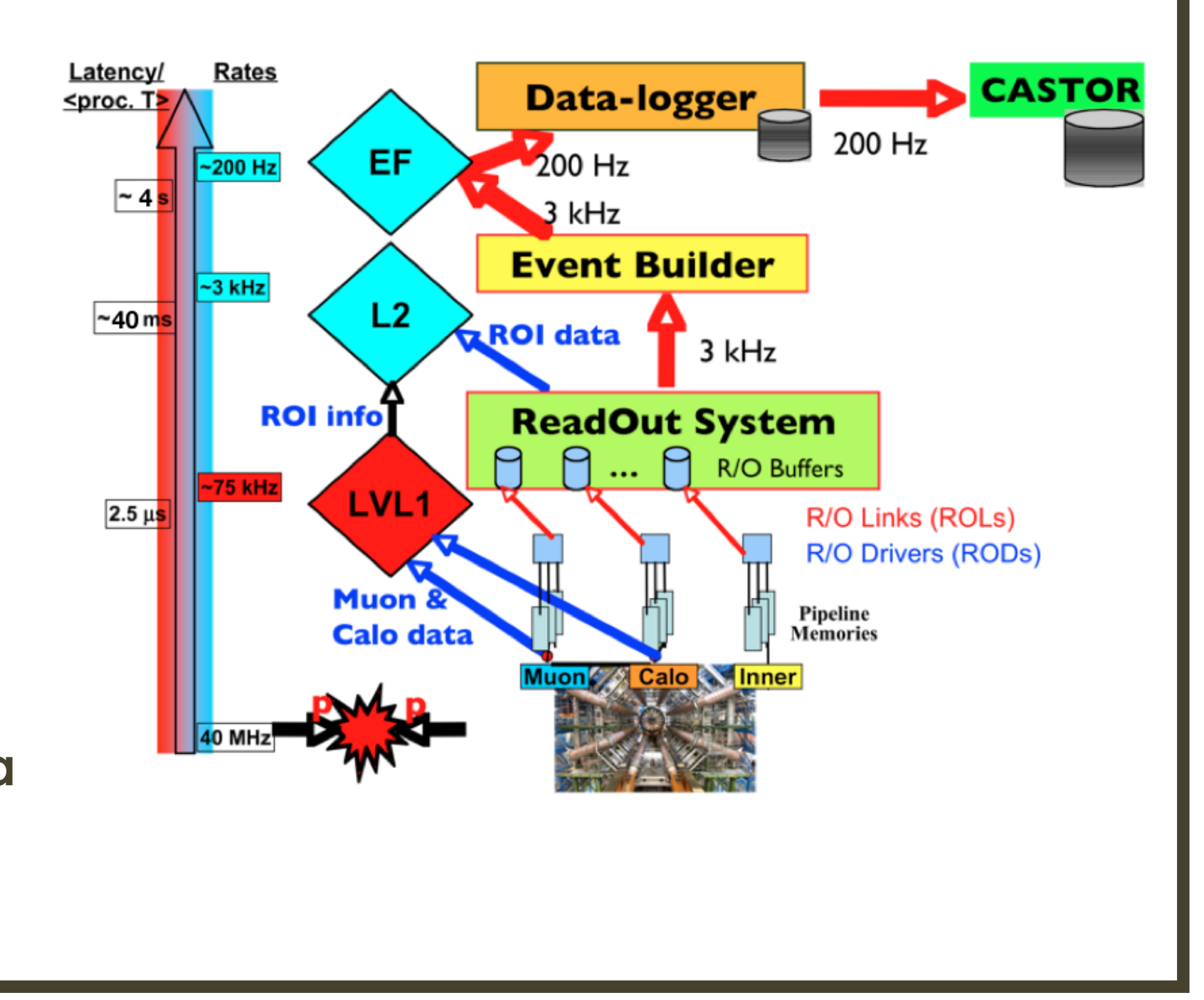

\section{Data Quality Monitoring Framework}

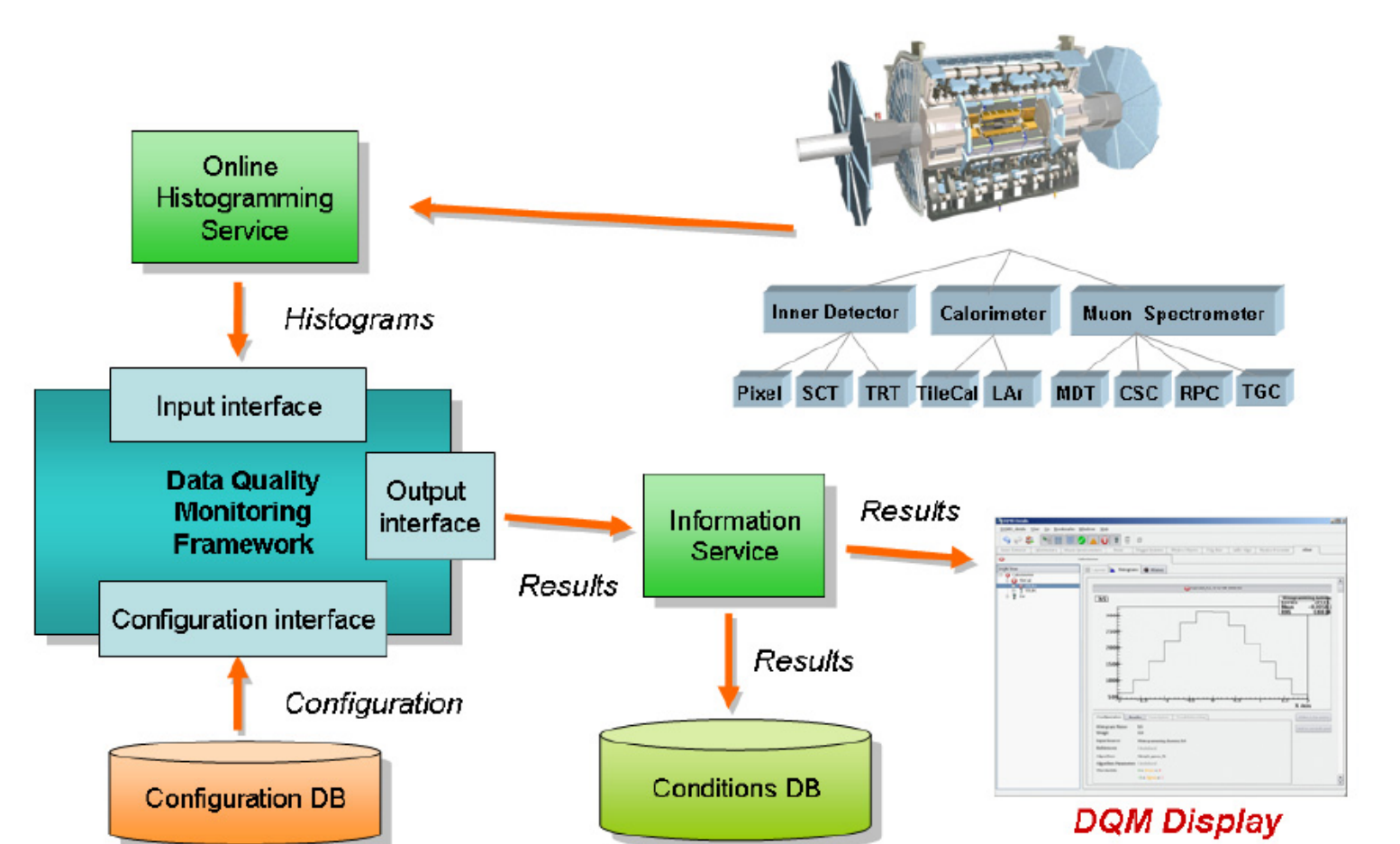

The Data Quality Monitoring Framework (DQMF) is a data driven distributed and scalable framework to monitor data quality both online and offline.

Main features of the framework

reading the configuration information from the data base

- finding and get all the available input histograms every time they are updated

- executing predefined tests, as specified in

the configuration

producing results and making them \$available

and writing to the DB

\section{Data Quality Monitoring Configurator}

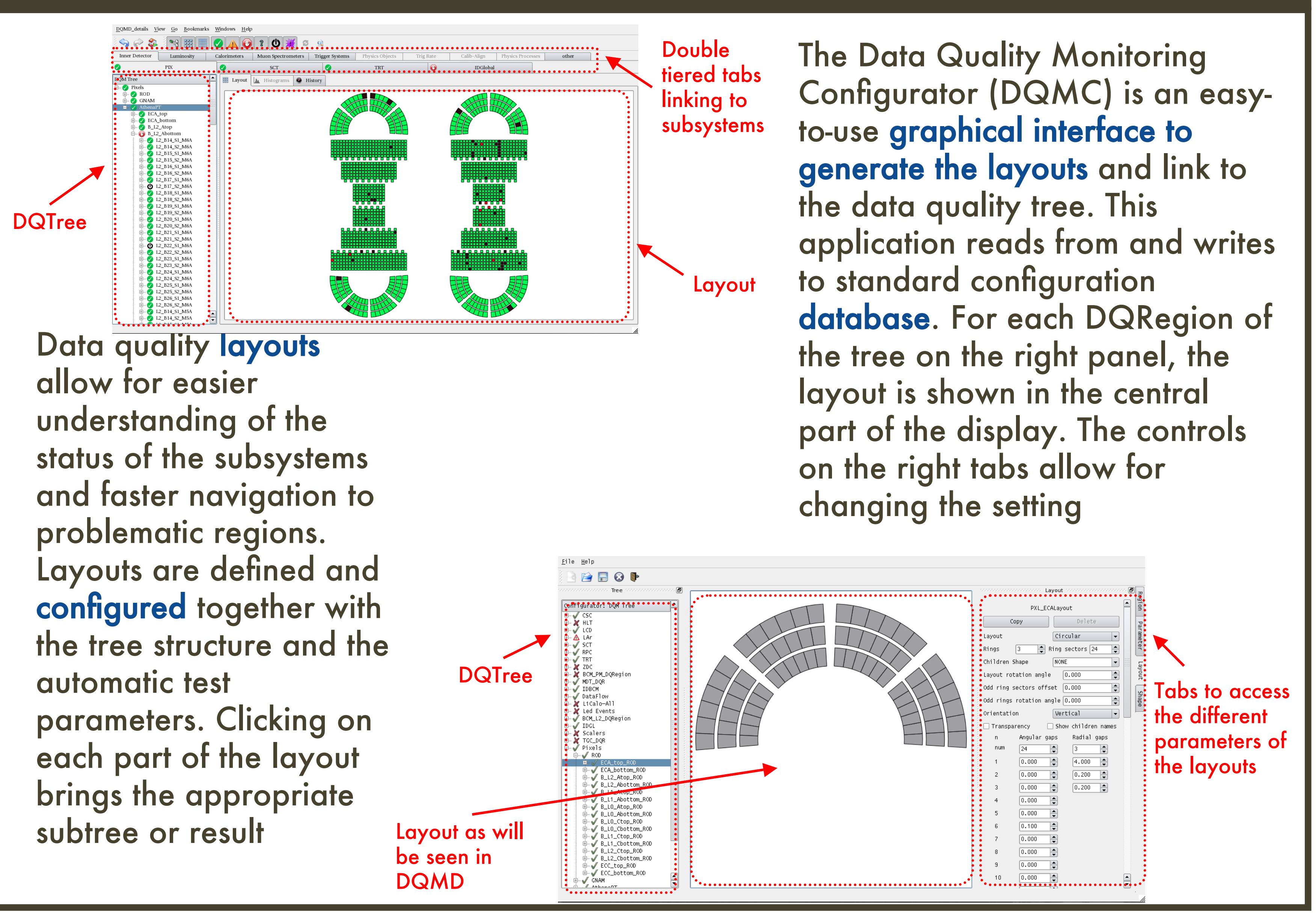

\begin{abstract}
DQTree
Single data quality (DQ) tests are handled by DQParameters. Each DQParameter specifies what input histogram(s) to use,
what algorithm (DQAlgorithm) to apply and the thresholds to classify the result (DQResult) as good or bad.

DQParameters are grouped in DQRegions. DQRegions also have DQResults associated. The mechanism to combine the resu configuration. DQRegions can be grouped in mother DQRegions, thus creating a $D Q$ tree.

DQAgent

$D Q$ agents are the applications at the core of the framework. These run the actual gorithms online. Input and output thus providing a large degree of flexibility. The checks, thresholds and parameters are loaded from the configuration data base.
\end{abstract}

\section{Monitoring infrastructure}

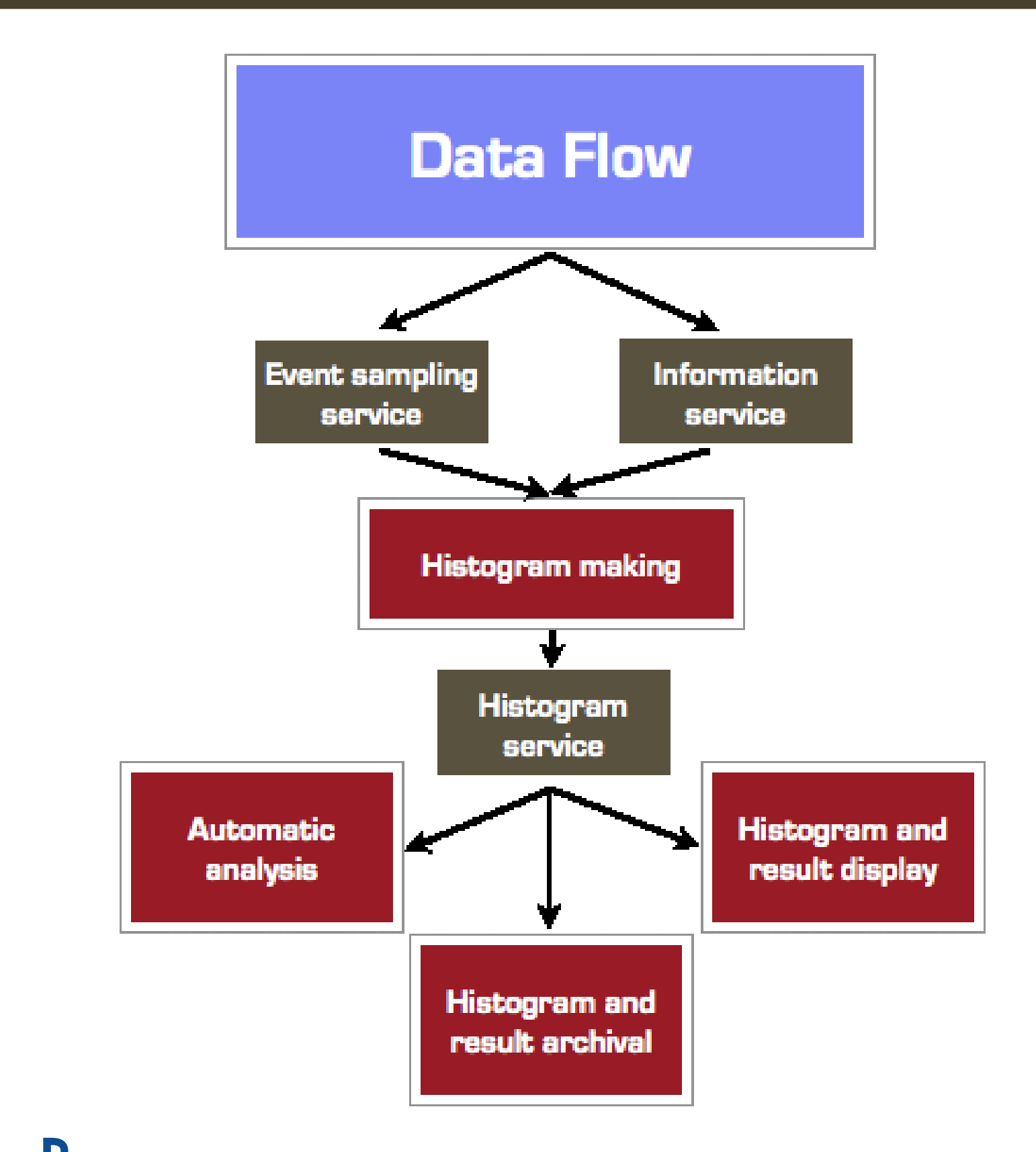

Resources

Monitoring uses about 32 dedicated machines. The different processes

access data and operational conditions at all stages of Data Flow and trigger analyze them and provide results.

\author{
A subset of the Data Acquisition system is \\ devoted to monitor the performance of each \\ element of the Data Flow and Trigger chains \\ and the quality of the data being recorded. \\ Monitoring main features \\ Many monitoring applications run online in \\ order to satisfy the large diversity of \\ monitoring needs: \\ monitoring needs: \\ Trigger and produce online histograms \\ - analyze operational conditions of the \\ different hardware elements \\ - perform automatic checks on the \\ histograms and operational data made \\ available by other applications \\ - archive all the data and results produced \\ online \\ - provide easy and fast visualization, locally \\ and remotely, of all the information made \\ available for shifters and experts.
}

